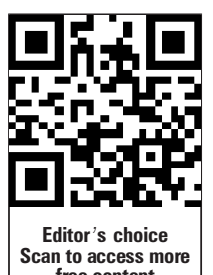

can to access mo free content

\section{Correspondence to} Dr Jane McVicar, Emergency Department, Royal Liverpool University Hospital, Prescot St Liverpool L7 8XP, Merseyside, UK; jane.mcvicar@rlbuht.nhs.uk

Accepted 29 April 2012 Published Online First 8 June 2012

\title{
Should we test for tetanus immunity in all emergency department patients with wounds?
}

\author{
Jane McVicar
}

\begin{abstract}
Background People who present to the emergency department often do not know their tetanus immunisation status. The Protetanus test can determine whether a person is immune and can guide treatment decisions.
\end{abstract}

Objectives To examine whether testing all patients for immunity is clinically advisable and cost-effective.

Methods 200 patients presenting to the emergency department with wounds were asked whether they were covered for tetanus, not covered or did not know. All had their tetanus immunity tested with the Protetanus kit and the result was compared with reported status. The number of unnecessary vaccinations which might have been given based on recall was calculated. The cost of testing and subsequent treatment versus vaccinations based on recall was calculated.

Results 136 (68\%) tested positive for tetanus immunity; $64(32 \%)$ were negative. Age, sex and nationality were not associated with tetanus immunity. 151 (75.5\%) did not know their status. Of these, 101 (66.9\%) were positive and $50(33.1 \%)$ negative. $49(24.5 \%)$ were sure of their status. 36 said they were covered of whom 10 $(27.8 \%)$ were wrong. 13 said they were not covered of whom 9 (69.2\%) were wrong. If vaccination were based on recall status $38.8 \%$ of patients would receive incorrect treatment. Testing all 200 and treating appropriately would save $£ 984$, or $£ 4.92$ per patient compared with vaccination based on recall.

Conclusion On clinical grounds testing is advisable and it may produce significant cost savings.

\section{INTRODUCTION}

Tetanus is a life-threatening disease caused by the bacterium Clostridium tetani which usually enters the body through an acute wound. Mortality is high $(20-50 \%)$ but it is now rare in the developed world because of preventive tetanus vaccination programmes. UK guidelines ${ }^{1}$ state that the five doses of the vaccine, scheduled between birth and school leaving age, should offer lifelong protection. However, it has been suggested that this immunity slowly declines over around 20 years $^{2}$ and that a further booster may be necessary. ${ }^{3}$ Also the UK schedule may leave older patients and some of those born outside the UK who did not receive a full course as children, unprotected. Importantly, when asked, a large proportion of patients simply do not know whether they have had five previous injections and often are unsure as to the date of their last one. When such patients attend the emergency department (ED) with an acute injury, they are often given tetanus boosters and the immediate but temporary protection of an immu- noglobulin injection 'to be on the safe side' Our department administers almost 3000 boosters every year and many of these may not be necessary. The Protetanus test kit can be used to determine whether an individual patient has a sufficient level of immunity to be protected against tetanus. It is used in Europe under the name Tetanos Quick Stick and has been validated against ELISA measurement of serum tetanus antitoxin level. ${ }^{4} 5$ It uses a single drop of blood from a finger prick and gives results in $10 \mathrm{~min}$. The manufacturer has a suggested protocol for its use (figure 1). We aimed to use the test on all patients attending the ED with an acute wound and compare their measured status with their recalled or reported status.

\section{METHODS}

The study was conducted at an urban ED in Merseyside. Written consent was obtained from all participants, and the study was approved by a local ethics committee.

We included consecutive patients aged 18 and over, attending with an acute wound. Exclusion criteria were age under 18 or inability for any reason to give consent.

Participants had their age, sex and origin recorded and they were asked to state their tetanus immunity status (immune, non-immune, do not know).

The definition of origin was kept very general (UK or non-UK) as the relatively small numbers involved would prohibit in-depth analysis of geographical effects.

The investigator assessed whether the wound was tetanus prone as defined by the Department of Health guidelines and documented their vaccination requirement based on recall (none, tetanus toxoid booster (TTB) or TTB and human tetanus immunoglobulin (HTIG)).

The Protetanus test was then performed on a fingerprick sample of the patient's blood and TTB and HTIG administered based on the measured result. A copy of the test result was sent to the GP and given to the patient on a small business card. Patients were advised to attend their GP for review if their primary course was thought to be incomplete and further boosters required.

The outcomes to be studied were: reliability of the patients' reported status; effect of age, sex and nationality on immune status; potential number of unnecessary vaccinations avoided by testing for immunity;

cost of universal testing versus vaccination based on recalled status-are there cost savings to be made by employing the test?

The effect of following the manufacturer's protocol for testing (figure 1). 


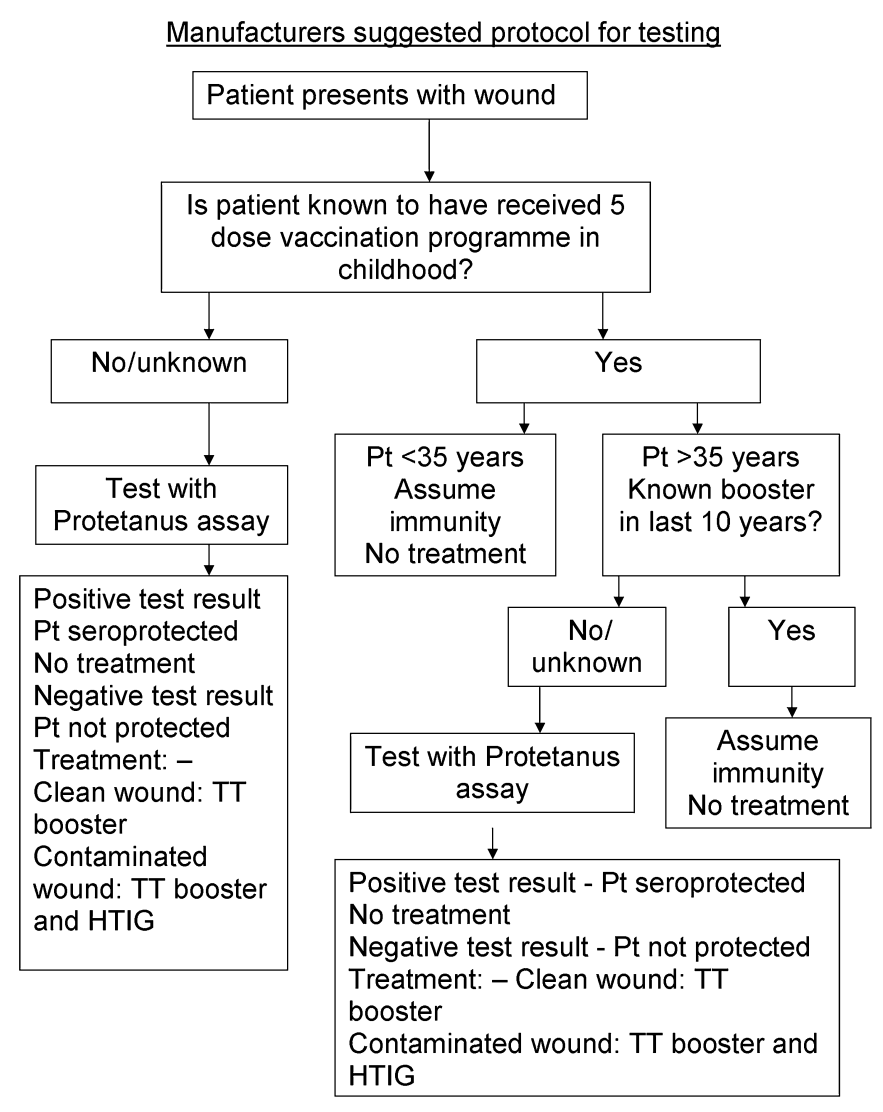

Figure 1 Manufacturer's suggested protocol. HTIG, human tetanus immunoglobulin; TT, tetanus toxoid.

Statistical advice was that a cohort of 200 patients would be sufficient to allow us to look at any relationship between simple variables such as age and sex and tetanus immunity status a $\chi^{2}$ test.

\section{RESULTS}

Two hundred and seven patients were recruited in 42 days, of whom seven were excluded. Three of these were aged $<18$ years, in two cases the paperwork was lost, one had insufficient blood for the test and one the blood was placed into the wrong well of the test cartridge. Two hundred patients were analysed (figure 2).

Mean age was 38.0 (18-94) with 131 (65.5\%) men and 69 (34.5\%) women - this was due to the majority of cases being recruited in the minor injury area of the department. Thirteen $(6.5 \%)$ patients described themselves as being born/brought up outside the UK.

Overall, 136 (68\%) tested positive for tetanus immunity and $64(32 \%)$ were negative-all of these were given TTB.

\section{Recall status}

One hundred and fifty-one (75.5\%) said they 'did not know' whether they were covered. Of these, 101 (66.9\%) tested positive and $50(33.1 \%)$ negative.

Forty-nine $(25 \%)$ were sure of their status. Thirty-six said they were covered, of whom $10(27.8 \%)$ were wrong. Thirteen said they were not covered, of whom nine $(69 \%)$ were wrong. If vaccination were based on recall status $38.8 \%$ of patients would receive incorrect treatment.

No relationship was found between sex $(p=0.11)$, non-UK origin $(p=0.55)$, age $(p=0.10)$ and immunity to tetanus.

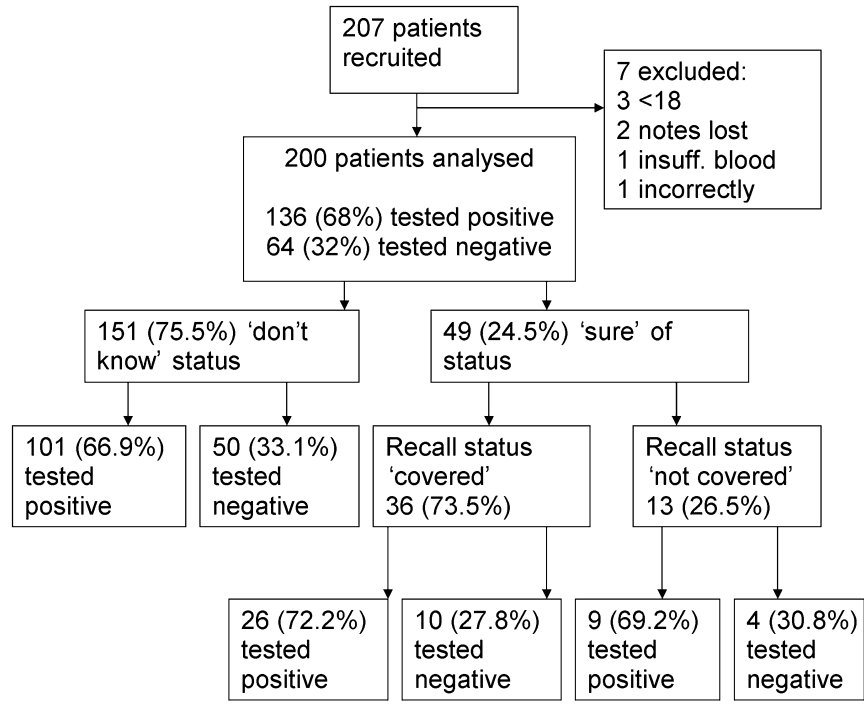

Figure 2 Results.

Ninety-one (45.5\%) wounds were judged to be tetanus prone. Fifty-five of these were among the immune patients and 36 were not immune; however, only three doses of HTIG were given.

Strategies:

cost of TTB-£6.74;

cost of HTIG- $£ 30$;

cost of test kit-£4.75.

\section{Vaccination based on patient's recall}

Assuming all unsure or not covered patients were given TTB and all tetanus-prone patients got HTIG:

TTB for all patients who were unsure or thought they were not covered $=(151+13) \times 6.74=£ 1105.36$

HTIG for patients who were unsure or not covered and tetanus prone $=78 \times 30=£ 2340$

Total $=£ 3445.36$

Also, 10 patients $(5 \%)$ who reported being immune but on testing were not, would miss their required TTB.

Vaccination based on testing all patients

The cost of test kits for all $200=£ 950$

TTB for all non-immune $=64 \times 6.74=£ 431.36$

HTIG for non-immune and tetanus prone $=36 \times 30=£ 1080$

Total $=£ 2461.36$

A saving of $£ 934.80$ or $£ 4.67$ per patient.

Vaccination based on manufacturer's protocol

Five non-immune patients who thought they were covered $(2.5 \%)$ would have been missed by using the protocol.

\section{DISCUSSION}

The study confirms previous findings that patient's recall of their immune status for tetanus is unreliable ${ }^{67}$ and this would seem to support the practice of testing all patients.

Testing all patients would mean that treatment was individualised and appropriate and the UK guidelines which have been shown to be poorly adhered to, ${ }^{8}$ could be significantly simplified.

Predicting the duration of immunity demonstrated by any one person's positive test is not possible and thus how often a test should be repeated is not clear at this stage. 
Universal testing appears to provide significant cost savings compared with vaccination based on recall, but these savings are unlikely to be quite so large in practice as they assume complete compliance with the national guidelines, which has been demonstrated not to be the case. We plan to introduce the test kits to our department and study the 'real-world' results.

An interesting finding was the low number of HTIG vaccinations given even when the person tested was judged by the investigator to have a tetanus-prone wound and shown to be non-immune-only $8 \%$ of those 'required' were given. This is similar to anecdotal figures from other hospitals in the UK (personal correspondence, Prospect Diagnostics) The staff are clearly making a clinical judgement about the degree of 'risk' the wound has which is not based on the national guidelines.

Despite this apparent lack of adherence to guidelines the incidence of tetanus remains low in the UK and the guidelines themselves could be called into question.

Use of the manufacturer's protocol would miss vaccinating a small group of young people $(2.5 \%$ of total) who were not immune as would be expected. This figure may be different in other areas, as Liverpool is known to have a poor take up of the fourth and fifth scheduled vaccinations. (personal correspondence, immunisation coordinator Liverpool 2010).

One strategy that might be of interest to ED physicians would be to completely ignore the tetanus status of any patients with non-tetanus prone wounds. These people have no significant risk of developing tetanus and any routine vaccinations that might be due are the responsibility of their primary care providers. Tetanus-prone wounds in the non-immune do constitute an 'emergency' and therefore identifying these people by testing and vaccinating as appropriate while referring the others to their GP may reduce the burden on EDs though further study would be necessary to look at cost-effectiveness. This policy might also have some impact on the population's immunity to diphtheria and polio as vaccines for both these diseases are given opportunistically in combination with the tetanus booster.

\section{Tetanus-prone wounds}

The guidelines for tetanus vaccination are contained in the green book. Tetanus-prone wounds are defined as:

wounds or burns that require surgical intervention that is delayed for more than $6 \mathrm{~h}$;

wounds or burns that show a significant degree of devitalised tissue or a puncture-type injury, particularly where there has been contact with soil or manure;

wounds containing foreign bodies;

compound fractures;

wounds or burns in patients who have systemic sepsis.

On reviewing the cases in the study the judgement of whether a wound was tetanus prone is hugely subjective, and in 38 cases it could be argued that the judgement was inaccurate on scrutinising the case notes. Rhee et al in their review in $2005^{10}$ suggest that evidence that more 'severe' wounds are tetanus prone is lacking and that clinical distinction between prone and non-prone wounds is not feasible.

The Department of Health was contacted for clarification as to what constitutes 'surgical intervention' and 'puncture-type injury'. They would not give any further information or direct evidence stating:

'There is no further guidance on what is meant by surgical intervention. Medical practitioners should take into account the likelihood that a wound is prone to infection by Clostridium tetani and treat accordingly' 'The advice given in the green book is based on expert medical opinion. Not all advice found in the green book can be linked to a particular study. As stated in our previous reply, we would recommend that clinical judgement be used when treating tetanus-prone wounds'.

\section{CONCLUSION}

On clinical grounds testing for immunity to tetanus is an appropriate strategy which avoids guesswork and unnecessary treatments. It may also reduce the cost of tetanus prevention to the ED.

Acknowledgements $E$ Caton, J Ellis, C Holt, M Hughes, G Murphy, G Murray, J O'Farrell and $\mathrm{H}$ Thackray for recruitment and data collection.

Competing interests Protetanus trial kits were provided free by Prospect Diagnostics. However, they had no part in the design, conduct or write up of the trial.

Ethics approval Provided by NW 6 REC.

Provenance and peer review Not commissioned; externally peer reviewed.

\section{REFERENCES}

1. Department of Health. Scottish Executive, Health Department, Welsh Assembly Government, DHSSPS (Northern Ireland). Tetanus. In: Salisbury DM, Ramsay ME, Moakes K, eds. Immunisation against infectious diseases - 'the green book'. London: TSO, 2006:367-84.

2. Simonsen 0, Bentzon MW, Kjeldsen $\mathrm{K}$, et al. Evaluation of vaccination requirements to secure continuous antitoxin immunity to tetanus. Vaccine 1987;5:115-22.

3. Cooke M. Are current UK tetanus prophylaxis procedures for wound management optimal? Emerg Med J 2009;26:845-8.

4. Colombet I, Saguez C, Sanson-Le Pors MJ, et al. Diagnosis of tetanus immunization status: multicentre assessment of a rapid biological test. Clin Diagn Lab Immunol 2005; 12:1057-62.

5. Elkharrat D, Sanson-Le Pors MJ, Arrouy L, et al. Evaluation of a bedside immunotest to predict individual anti-tetanus seroprotection: a prospective concordance study of 1018 adults in an emergency department. Emerg Med J 2010;27:36-42.

6. Fishbein DB, Willis BC, Cassidy WM, et al. Determining indications for adult vaccination: patient self-assessment, medical record or both? Vaccine 2006;24:803-18

7. Gindi $\mathbf{M}$, Oravitz $\mathrm{P}$, Sexton $\mathrm{R}$, et al. Unreliability of reported tetanus vaccination histories. Am J Emerg Med 2005;23:120-2.

8. Talan DA, Abrahamian FM, Moran GJ, et al. Tetanus immunity and physician compliance with tetanus prophylaxis practices among emergency department patients presenting with wounds. Ann Emerg Med 2004;43:305-14.

9. Savage EJ, Nash S, McGuiness A, et al. Audit of tetanus prevention knowledge and practices in accident and emergency departments in England. Emerg Med $\mathrm{J}$ 2007:24:417-21

10. Rhee $\mathbf{P}$, Nunley $M$, Demetriades $D$, et al. Tetanus and trauma: a review and recommendations. J Trauma 2005;58:1082-8. 